\title{
Towards a Wave Theory Interpretation of Time-Distance Helioseismology Data
}

\author{
A. C. Birch
}

Department of Physics, Stanford University, Stanford, CA 94305

\author{
A. G. Kosovichev \\ W.W. Hansen Experimental Laboratory, Stanford University, Stanford, \\ CA $94305-4085$
}

\begin{abstract}
Time-distance helioseismology, which measures the time for acoustic waves to travel between points on the solar surface, has been used to study small-scale three-dimensional features in the sun, for example active regions, as well as large-scale features, such as meridional flow, that are not accessible by standard global helioseismology. Traditionally, travel times have been interpreted using geometrical ray theory, which is not always a good approximation. In order to develop a wave interpretation of time-distance data we employ the first Born approximation, which takes into account finite-wavelength effects and is expected to provide more accurate inversion results. In the Born approximation, in contrast with ray theory, travel times are sensitive to perturbations to sound speed which are located off the ray path. In an example calculation of travel time perturbations due to sound speed perturbations that are functions only of depth, we see that that the Born and ray approximations agree when applied to perturbations with large spatial scale and that the ray approximation fails when applied to perturbations with small spatial scale.
\end{abstract}

\section{Introduction}

Local helioseismology is important as it allows the study of three dimensional subsurface flows and structures. The time-distance method, pioneered by $\mathrm{Du}$ vall and coworkers (e.g. Duvall et al., 1993; Kosovichev \& Duvall, 1997) uses cross-correlations between the oscillation signals at different points on the surface to infer the time for an acoustic wave packet to travel between those points. Numerous studies have employed the time-distance method to probe flows and structures that are inaccessible using traditional global helioseismology (e.g. Duvall et al., 1997; Giles, 1999 ).

Inversions of time-distance data, inferences of flows and structure perturbations from travel times, are typically done in the ray approximation (e.g. Kosovichev \& Duvall, 1997). In the ray approximation, travel time perturbations are expressed as integrals of perturbations along the geometrical ray path connecting the two points the travel time is measured for. Ray theory is not, 

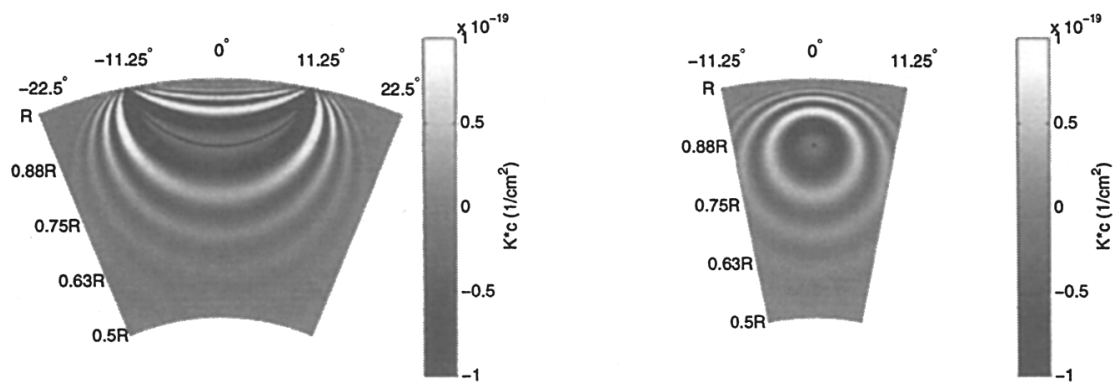

Figure 1. Two slices through a Born approximation travel time kernel. The left panel shows a cut in the plane of the ray path. The black line is the ray path. The right panel is a cut in the plane perpendicular to the ray path at the lower turning point.

however, expected to accurately represent the dependence of travel times on perturbations with small spatial scale (e.g. Bogdan, 1997; Jensen et al., 2000). The first Born approximation is an appealing alternative to the ray approximation as it includes finite-wavelength effects and has been explored in some detail in the geophysics literature (e.g. Zhao et al., 1998; Marquering et al., 1999). We have begun to apply the Born approximation to time-distance helioseismology (Birch \& Kosovichev, 2000).

\section{Comparison Between the Born and Ray Approximations}

Figure 1 shows two slices through a Born approximation travel time kernel, calculated in the model of Birch \& Kosovichev (2000), which gives the sensitivity of travel time to sound speed perturbations, for points separated by a distance of $22.5^{\circ}$. In the ray approximation the travel time kernel is proportional to a delta function along the ray path, which is the black line in Figure 1. As has been seen in the geophysics literature, in the Born approximation, travel times are most sensitive to sound speed perturbations on a hollow cylinder surrounding the ray path (e.g. Marquering et al., 1999).

Figure 2 shows two series of calculations of travel time perturbations. For perturbations with large spatial scale, the right hand panel, the Born and ray approximations agree. In the left panel the spatial scale of the sound speed perturbation is reduced by a factor of ten and the two approximations disagree. The Born approximation, however, agrees with standard normal mode perturbation theory for the case of spherically symmetric sound speed perturbations (Birch \& Kosovichev, 2001).

\section{Discussion}

We have shown an example travel time sensitivity kernel in the Born approximation and demonstrated, via a series of forward calculations, differences between travel time perturbations calculated using the Born and ray approximations. The ray approximation does not work well when applied to perturbations with sufficiently small spatial scale. 

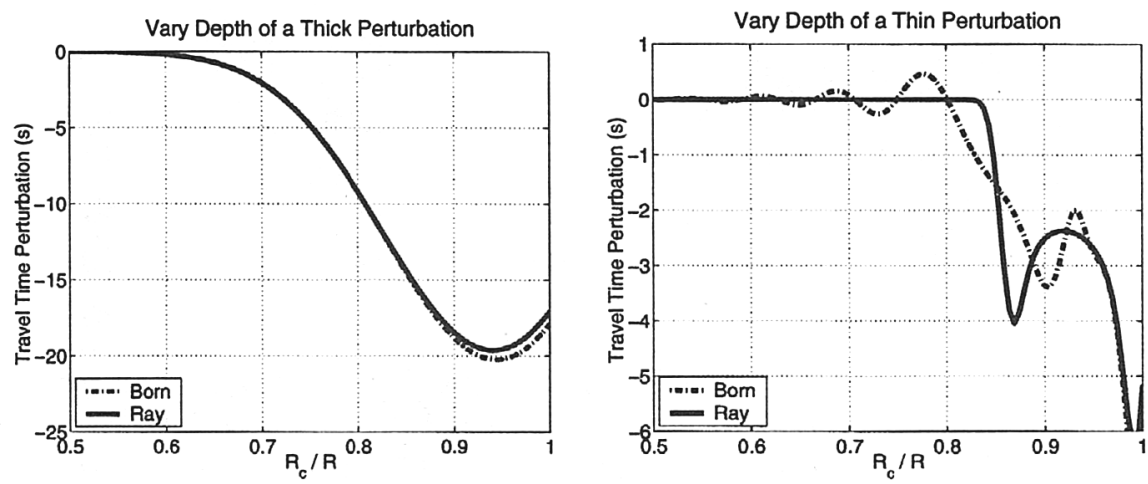

Figure 2. Travel time perturbations due to sound speed perturbations in the ray and Born approximations. The left panel is for sound speed perturbations with Gaussian dependence on radius, with amplitude of $1 \%, \mathrm{FWHM}$ of $0.24 R_{\odot}$, and varying central radius, $R_{c}$. In the right panel the FWHM is reduced to $0.024 R_{\odot}$.

The failure of the ray approximation in some of the example calculations does not, however, mean all previous ray theory inversion results should be discarded. Inversions involve averages over many rays; a more detailed look at the implications of using Born kernels in place of ray kernels is needed. We plan to calculate sensitivity kernels for flows and to do three dimensional inversions using Born kernels. This work was supported by NASA grant NAG5-8878.

\section{References}

Birch, A. C. \& Kosovichev, A. G. 2000, Solar Phys., 192, 193

Birch, A. C. \& Kosovichev, A. G. 2001 in: Proc. SOHO 10 / GONG 2000 Conference, Helio- and Asteroseismology at the Dawn of the New Millennium, ed. A. Wilson, et al., ESA Publication SP-464, in press

Bogdan, T. J. 1997, ApJ, 477, 455

Duvall, T. L. Jr., Jefferies, S. M., Harvey, J. W., \& Pomerantz, M. A. 1993, Nature, 362,430

Duvall, T. L. Jr., et al. 1997, Solar Phys., 170, 63

Giles, P. M. 1999, Time-Distance Measurements of Large-Scale Flows in the Solar Convection Zone, Ph.D. thesis, Stanford University

Jensen, J. M., Jacobsen, B. H., \& Christensen-Dalsgaard, J. 2000, Solar Phys., 192,231

Kosovichev, A. G. \& Duvall, T. L. Jr. 1997 in SCORe'96: Solar Convection and Oscillations and their Relationship, ed. J. Christensen-Dalsgaard \& F. Pijpers (Aarhus: Kluwer), 241

Marquering, H., Dahlen, F. A., \& Nolet, G. 1999, Geophys. J. Int., 137, 805

Zhao, L. \& Jordan, T. H. 1998, Geophys. J. Int., 133, 683 